\title{
Auction-based Crossings Management
}

\author{
Giacomo Cabri \\ Dip. Scienze Fisiche, \\ Informatiche e Matematiche \\ Univ. Modena e Reggio Emilia \\ Modena, Italy \\ giacomo.cabri@unimore.it
}

\author{
Luca Gherardini \\ Dip. Scienze Fisiche, \\ Informatiche e Matematiche \\ Univ. Modena e Reggio Emilia \\ Modena, Italy \\ 218121@studenti.unimore.it
}

\author{
Manuela Montangero \\ Dip. Scienze Fisiche, \\ Informatiche e Matematiche \\ Univ. Modena e Reggio Emilia \\ Modena, Italy \\ manuela.montangero@unimore.it
}

\begin{abstract}
Autonomous vehicles will "invade" our street in a not so far future. They must be coordinated in order to exploit the resources in a fair yet effective way. One of these resources, whose management is quite challenging, is represented by crossings: vehicles come and aim at passing the intersection, often as soon as possible, but they must compete with other vehicles.

In this paper we propose an auction-based mechanism to coordinate the vehicles at a crossing, considering the presence of both human-driven and autonomous vehicles; moreover, we introduce an enhancement mechanism to take into consideration also the presence of vehicles in the lanes behind the first one. We analyze different strategies and, by means of simulation experiments, we compare them and show how waiting times change depending on the strategies. The results show that the cooperative strategy presents average waiting times lower for all vehicles than a competitive strategy.
\end{abstract}

\section{CCS CONCEPTS}

- Computing methodologies $\rightarrow$ Cooperation and coordination; Self-organization.

\section{KEYWORDS}

Autonomous vehicles, coordination, auction

\section{ACM Reference Format:}

Giacomo Cabri, Luca Gherardini, and Manuela Montangero. 2019. Auctionbased Crossings Management. In EAI International Conference on Smart Objects and Technologies for Social Good (GoodTechs '19), September 25-27, 2019, Valencia, Spain. ACM, New York, NY, USA, 6 pages. https://doi.org/10. $1145 / 3342428.3342689$

\section{INTRODUCTION}

With the rapid growth o the IoT [6] and new opportunities of making almost everything smart, smart mobility [7] will improve our experience of driving in smart cities: our streets will be soon populated by autonomous vehicles, which will take autonomous decisions about the route to follow, not only considering the fastest

Permission to make digital or hard copies of all or part of this work for personal or classroom use is granted without fee provided that copies are not made or distributed for profit or commercial advantage and that copies bear this notice and the full citation on the first page. Copyrights for components of this work owned by others than ACM must be honored. Abstracting with credit is permitted. To copy otherwise, or republish, to post on servers or to redistribute to lists, requires prior specific permission and/or a fee. Request permissions from permissions@acm.org.

GoodTechs '19, September 25-27, 2019, Valencia, Spain

(c) 2019 Association for Computing Machinery.

ACM ISBN 978-1-4503-6261-0/19/09 . \$15.00

https://doi.org/10.1145/3342428.3342689 path between the origin and the destination, but also considering the cost of the route, the needs of the driver, the current traffic, and so on.

However, smart mobility must be supported. In particular, crossings represent a challenge [2]: the traditional traffic lights are not a suitable mechanism, because of its rigidity in assigning resources (the chance of pass the crossing), and the presence of autonomous vehicles imposes to consider a dynamic resource allocation. Moreover, the presence of also human-driven vehicles must be taken into consideration.

In the past, some approaches were proposed to address collaborative management of intersections [5, 10]. Often, auctions are exploited to dynamically manage resources (e.g., see [4] ), because they enable to define the price of a good on the base of customer interest [1]. Auctions were largely exploited to manage negotiations between autonomous entities modelled as agents [3]. The main weaknesses of the existing approaches are that they consider autonomous vehicles only and do not compare different strategies.

In this paper we present some preliminary experiments related to a longer term project in which we will analyze the benefits of exploiting auctions in managing vehicles at crossings. Indeed, the scenario we set up has some differences with respect to previous work. In particular, we consider the presence of both autonomous vehicles and human-driven vehicles, we propose different strategies both competitive and collaborative, and we define an enhancement mechanism that takes into consideration in the auction process also the number of vehicles in the lanes, in order to speed up long lanes. Our long-term aim is to understand which, among possible different choices, leads to better traffic management.

We are aware that sometime in the future we are likely to have only autonomous vehicles, but we think that there will be a transient period in which human-driven vehicles will co-exist, and an appropriate management of this period will leverage the adoption of autonomous vehicles.

The paper is organized as follows. We first present and discuss some related work (Section 2), then, we present the approach we propose to manage vehicles at crossings (Section 3). The experimental results, obtained by means of the SUMO (Simulation for Urban MObility) simulator, are shown and discussed in Section 4. Finally, we conclude the paper proposing some future work (Section 5).

\section{RELATED WORK}

We present auction-based approaches for crossing management (Section 2.1) and approaches for general coordination of autonomous vehicles (Section 2.2). 


\subsection{Auctions at Intersections}

In one of the milestone papers exploiting auctions in crossings management [4], authors address the value of time of each vehicle, representing it by means of a wallet system for automatic bidding based on trip characteristics, driver-specified budget, and remaining distance to the destination. Moreover, they address also the optimization of the overall traffic. The main differences with our work are: first, we consider both autonomous vehicles and human-driven vehicles; second, we propose different strategies both competitive and collaborative; third, we enable to apply an enhancement that takes into consideration the number of vehicles in the lanes.

Vasirani and Ossowski [12] propose an approach to manage urban crossings based on market-inspired rules. Differently from that approach, we do not assume that all vehicles are autonomous, considering also human-driven vehicles.

Schepperle and Böhm [11] proposed a two-step auction mechanism to manage vehicles at intersections, based on a second-price sealed-bid auction [13]: in the former step only vehicles that can pass the intersections are involved, while in the latter step also some vehicles in the second place of the lane can bid, depending on the result of the first step for the preceding vehicle. Even if that approach was quite effective, we decided to define a simpler approach, in order to consider the total number of vehicles in line to enhance the bid of the vehicle in the front.

\subsection{Coordination of Autonomous Vehicles}

The work in [9] addresses the programming of autonomous robots by pointing out the difference between smart devices and autonomous vehicles in a navigation problem; the formers have little capability of interacting with the physical world, while the robots in general and autonomous vehicles in particular rely on sensors and actuators to interact and move in the physical environment. They propose a swarm language construct that enables to categorize robots in swarms and assign tasks to the swarms. Their goals are re-usability and predictability of the coded behavior, two very important features in the field of autonomous driving.

An example of such goals can be inferred also from [8], in which a simulated environment is designed to have cars traveling in a highway, self-organize themselves in platoons with the final goal to reduce fuel consumption, by drafting off one another.

More recently, the CLASS Horizon 2020 project (https://classproject.eu/) has the goal to instrument a one square Kilometer-wide area in the city of Modena (Italy) with a heavy sensor infrastructure with the purpose to collect and process in real-time the resulting vast amount of data. Such data will be used to communicate to the connected cars. Such cars are equipped with heterogeneous sensors/actuators and V2X connectivity so to enhance both driving experience and city overall safety. This is achieved by deploying advanced urban mobility applications based on a combination of data-in-motion and data-at-rest analytics to efficiently coordinate cars and city computing resources. As of now, the part of the city covered by cameras and sensors is depicted in Figure 1.

\section{THE PROPOSED APPROACH}

In this section we explain our approach, detailing the assumptions, the management of bids, and the evaluated strategies.

\subsection{Assumptions}

In our scenario we assume that autonomous vehicles and humandriven vehicles coexist and that all vehicles are equipped to participate to the crossing management mechanism. In particular, humandriven vehicles do not require humans to directly intervene, but they will be equipped with a device that is autonomously able to participate to the mechanism. We assume that each crossing has its own management system that is run on a physical device that is placed at the crossing site. The vehicles crossing priorities are decided by the crossing management system by means of an auction: vehicles at the front of a lane make their own bid according to their urgency to pass the crossing and to their budget.

\subsection{Bids}

Bids are set using a virtual coin and, as a vehicle joins the system, it gets a fixed amount of virtual coins. If a vehicle runs out of virtual coins, the system will let it make a minimal bid anyway. In our experiments, we do not concern about how vehicles get the money to bid, so we exploited a simple mechanism to "recharge" the virtual wallet in order to enable vehicles to bid. In real scenarios, virtual coins might be implemented according to different policies: they might be bought with real money, or, for example, might reflect the drivers (good or bad) behaviour while driving.

\subsection{Strategies}

We devise two different approaches for auction resolution:

- Cooperative approach (COOP): all the vehicles at the front of the lanes at the crossing make their bid, and all bidding vehicles will go through the crossing according to the bid order (highest fist).

- Competitive approach (COMP): all the vehicles at the front of the lanes at the crossing make their bid, but only the vehicle that wins the auction gets to pass the crossing. Vehicles that lost the auction will have to attend and win a successive auction before being able to go through the crossing. Note that this may imply starvation.

Moreover, we consider two different methods for the bid payment:

- All-Pay (AP): All bidding vehicles are charged for their bid.

- Only-Winner-Pays (OWP): only the vehicle that wins the auction will be charged for its bid, while vehicles that lost the auction are not charged.

In our experiments we will investigate the effectiveness of the four strategies arising from all possible combinations of approaches and paying methods. We observe that the strategies COOP-AP and COMP-OWP seems the most natural to adopt, and are complementary: in the former all vehicles have to pay their bid, but they are sure to go through the crossing after the auction is over, even if they do not know in which order. This round-robin like approach guarantees that all lanes proceed at each auction round. In the latter, on the contrary, lanes with more aggressive auctioneers might make one lane move faster than the others. The other two strategies, namely COOP-OWP and COMP-AP, seem unusual but we think that they are worth investigating anyway. 

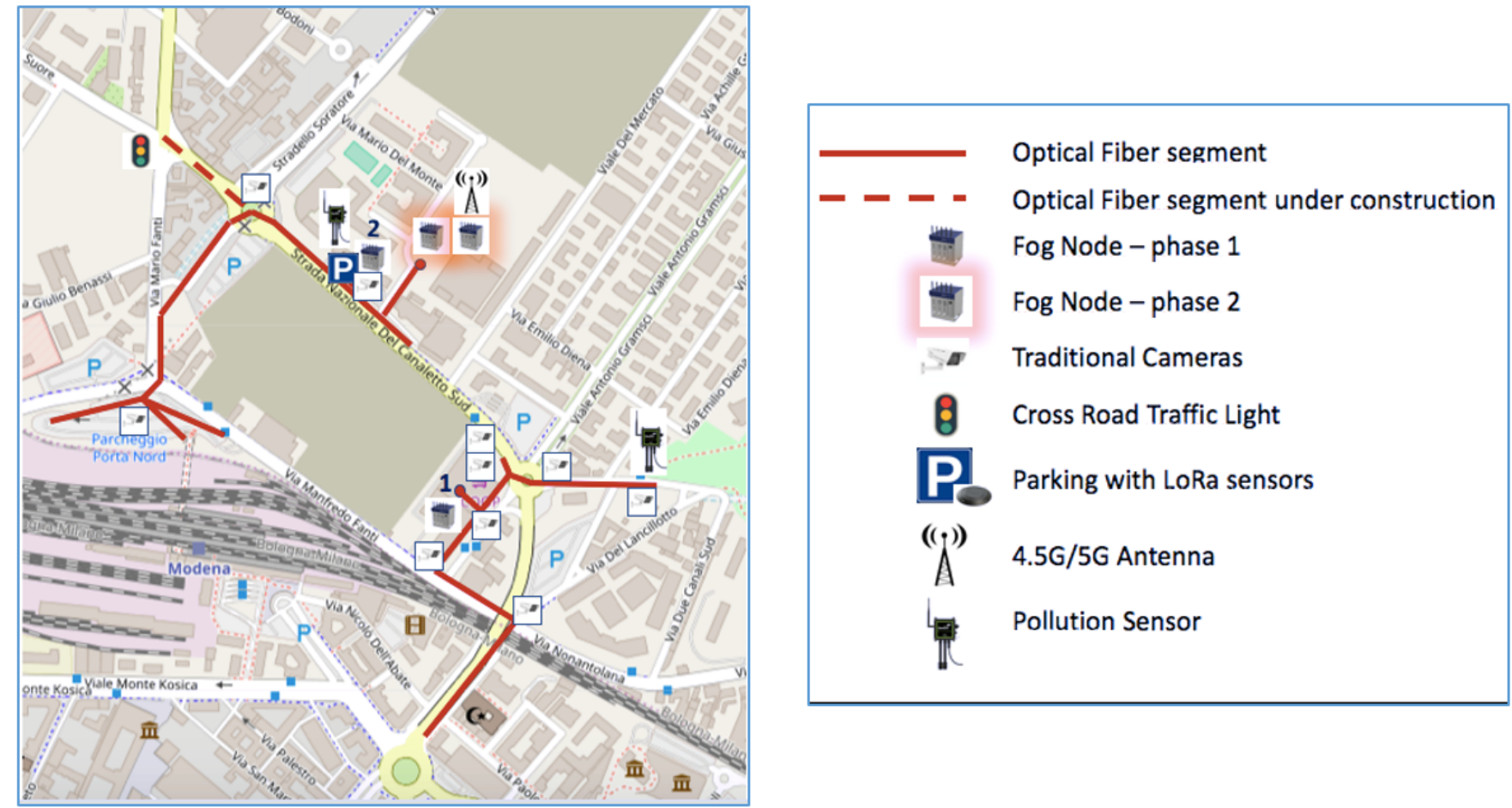

Figure 1: Modena Automotive Smart Area.

3.3.1 Enhancement. We devise an enhancement mechanism that is meant to balance the waiting times of lanes with long lines with respect to lanes with a smaller number of vehicles in line. To this aim, we "adjust" the front lane bidding according to the number of cars waiting in the same lane. In particular, for each lane $\ell$ with $n_{\ell}$ cars in line and bid $b_{\ell}$ made by the car at the front of the lane, we formally define the enhancement in the following way:

$$
e n(\ell)=b_{\ell}\left(\ln n_{\ell}+1\right)
$$

The value $e n(\ell)$ is the actual bid for the front vehicle of lane $\ell$. Observe that $e n(\ell)=b_{\ell}$ when the only vehicle in the lane is the front one, while the logarithmic multiplicative factor gets the more significant the larger the number of vehicle waiting in the lane.

\section{EXPERIMENTS}

In this section we explain how the experiments were performed and we report about the results.

\subsection{Implementation}

Algorithms have been implemented in Python using the SUMO (Simulation for Urban MObility) simulator ${ }^{1}$ and its API. SUMO gives the possibility to define: i) a road map as a graph, by specifying roads as edges and lanes within roads; ii) vehicles with desired behaviour.

Figure 2 shows the SUMO road map that has been used in the experiments presented in this paper. Figure 3 shows a close-up of

\footnotetext{
${ }^{1}$ https://www.dlr.de/ts/en/desktopdefault.aspx/tabid-9883/16931_read-41000/
}

an intersection with eight vehicles at a cross road in which it is possible to distinguish road lanes.

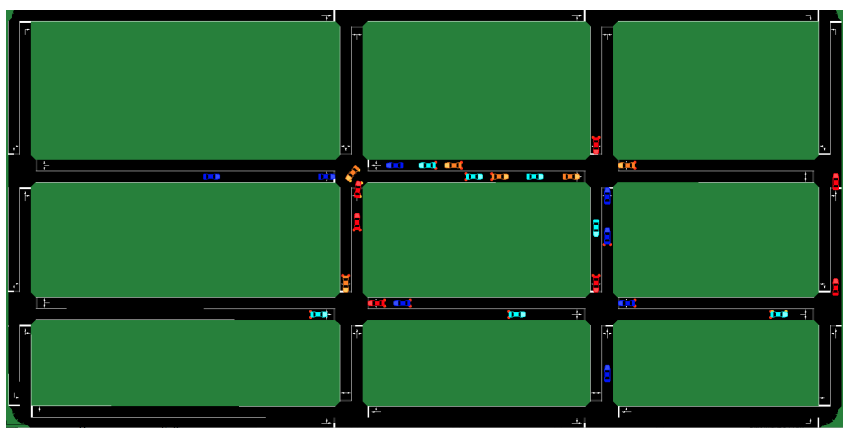

Figure 2: Road map used in the experiments.

\subsection{Experimental set up}

Vehicles are characterized by the following parameters:

- A driving mode: might be autonomous or human-driver. Such mode has no influence on the bidding mechanism, but it influences the responses time of vehicle to events. In particular, human-driven vehicles take a longer time to leave the crossing when it is their turn, influencing the waiting time of the other vehicles at the crossings and indirectly the waiting time of vehicles in the lanes. 


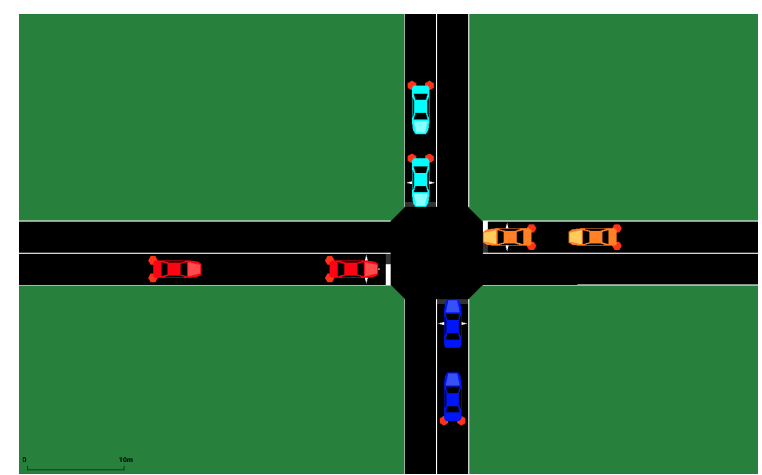

Figure 3: Map close-up at intersection.

- A budget: initially set to a default value, and regularly updated after each auction the vehicle is involved in. As we said, in these experiments we implement a recharging budget strategy that, after each auction, simply equally distributes the paid bids to all bidding vehicles.

- A degree of interest in passing the crossing as soon as possible, which influences the amount of the bids. Since it depends on different factors not related to the approach, in our simulations we have chosen random bids within the budget range.

- A route: a route is a circular path in the road map, starting from an edge and returning to the same edge. Once a vehicles returns to the starting edge it can be assigned to the same route again (static route), or a different one randomly starting from the edge the vehicle is currently on (random route).

- An identifier: a unique ID to distinguish vehicles.

Each experiment is characterized by its own scenario, defined by selecting one approach for auction resolution (COOP or COMP), one payment method (AP or OWP), enabling or disabling enhancement (E or NE), static or random routes for vehicles (SR or RR). In this preliminary study, for each experiment, we have that all vehicles behave according to the selections in the scenario.

We run experiments for all possible scenarios, once with 30 vehicles and once with 75 , half human-driven and half autonomous in all cases, to test different traffic conditions. Waiting times are not measured in seconds, but rather in SUMO simulation steps (to be independent of particular situations occurring on the computer performing the simulation, e.g., other processes running during part of the simulation and influencing the times). Simulations are run for 5000 time steps each.

For each experiment, we report the following measures:

- Statistics on vehicles average waiting times to clear the crossings: we compute the average time each vehicle spends waiting at a crossing before going through and report the average, maximum, minimum, and standard deviations of such averages.

- Statistics on average times vehicles spend waiting in lanes: compute the average time each vehicle spends waiting in lane and then report the average, maximum, minimum, and standard deviations of such averages.
- Number of times in which a vehicles finds an empty lane to get to the crossing, given as the percentage over the total number of crossing traversing during the simulation.

\subsection{Experimental Results}

In this section we report the results of the experiments conducted as explained in the previous section. We show results only for the static route selection, for lack of space and because there are no significant differences in the results of the two different choices. Such a difference will be taken into consideration in further, more fine-grained analysis involving more experiments.

Some results confirm some a-priori intuitions (e.g., cooperative approach should be fairer than the competitive one), but there are also unexpected results, some of which will be the focus of further investigation in future work to be better understood. We present agglomerate results for human-driven and autonomous vehicles, as the difference of waiting times, on average, is at most one time step.

4.3.1 Average time to clear the crossing. Statistics are shown in Figure 4. As it was to be expected, on average, waiting time is smaller in the cooperative approach than in the competitive one. This is due to the fact that at the end of each auction round in the former approach all vehicles at the front of the lanes go through the crossing, while in the latter all but one have to wait for the next auction. On the other side, we observe that, within each approach, average waiting time does not change much, as well as with different number of vehicles. Indeed, in the competitive approach average waiting time is between 11 and 12.5 time steps, and in the cooperative is between 4 and 4.8 time steps; for 75 vehicles it is between 11 and 13.2 for the competitive approach, and between 2.8 and 3.8 in the cooperative approach. The fact that the cooperative approach seems to perform better with heavier traffic was, on the contrary, unexpected. One possible explanation is that the time to clear the crossing depends also on the vehicles trajectories. For example, two vehicles might traverse at the same time if their trajectories do not intersect, otherwise one has to wait until the other completely frees the crossing before start moving. The actual time for four vehicles to completely clear the crossing goes from a minimum of four to a maximum of six time steps, therefore, such small differences might just be caused by the specific trajectories happening in the different simulations. We will however deeply investigate this result in our future work.

With regard to the paying method, we can point out that, for both approaches, OWP leads to more balanced and uniform results in terms of times than AP: max values are smaller, min values are larger, standard deviations are smaller. This is due to the fact that only the winning vehicle pays and this leads to an unbalance in terms of budget that gives advantage to the vehicles that lost the auction, giving them more chances to win the next auctions. This phenomenon is probably emphasized by our budget redistribution policy by which the winning bid is redistributed among all participants to the auction. On the contrary, in the AP approach we have a more balanced situation in terms of budget, because all vehicles involved in the auction pay (and the total amount is redistributed). This leads to situations in which budget is uniformly distributed and it does not give much advantage to the vehicles that have lost 


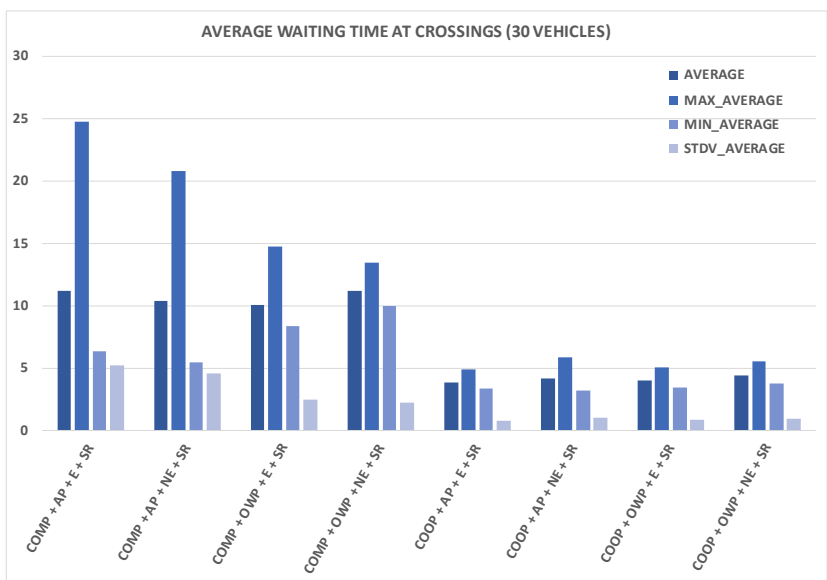

(a) 30 vehicles

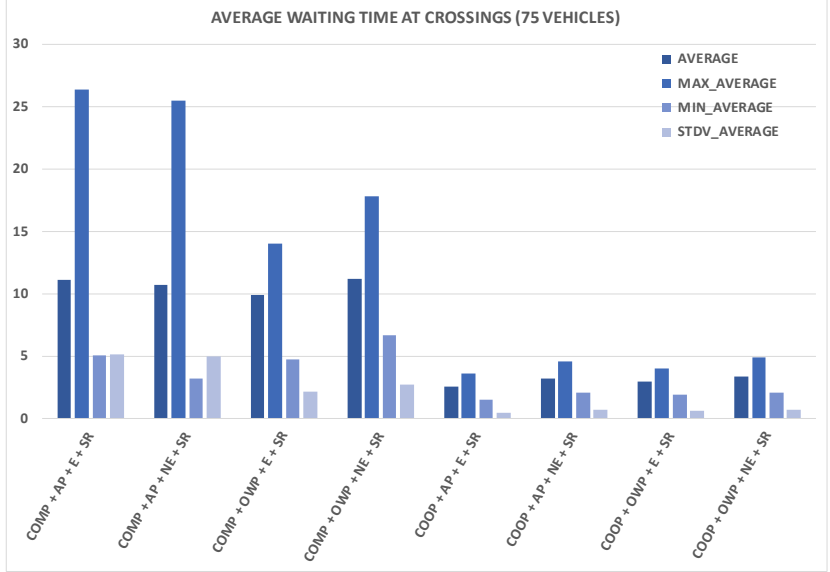

(b) 75 vehicles

Figure 4: Average waiting time to clear crossing statistics.

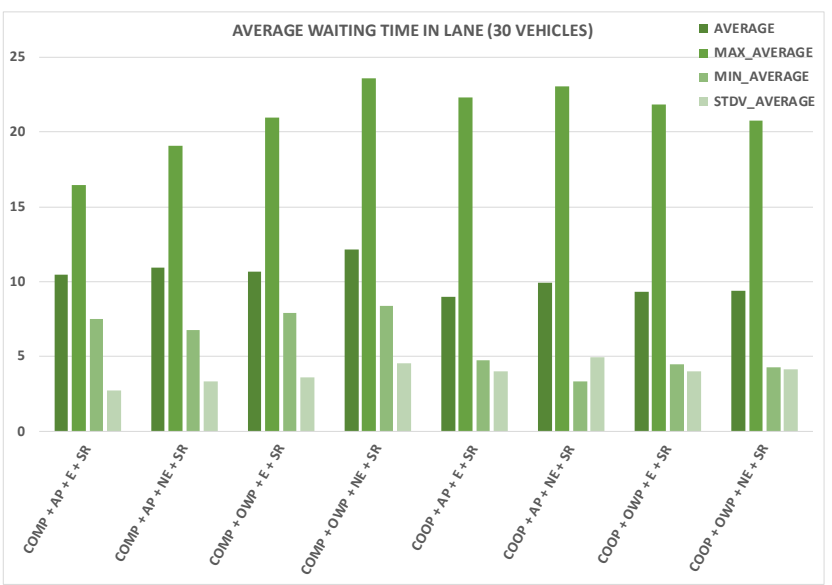

(a) 30 vehicles

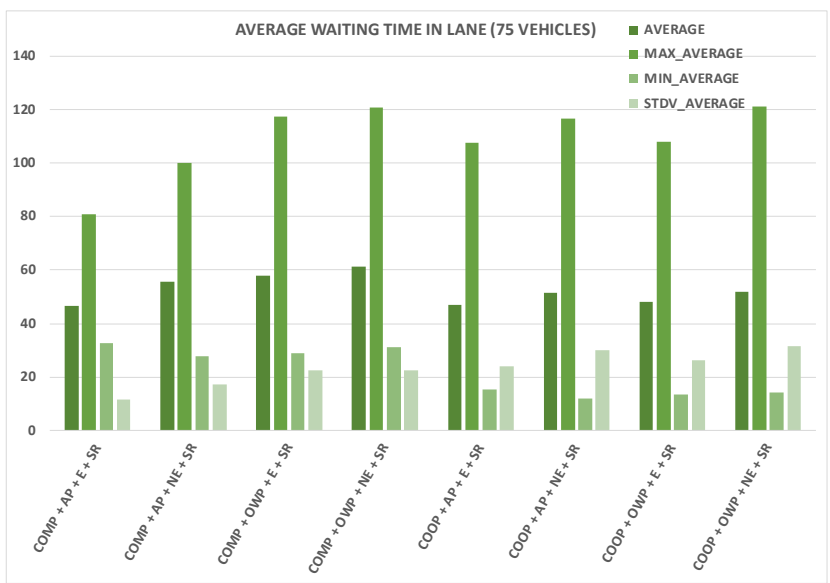

(b) 75 vehicles

Figure 5: Average waiting times in line statistics

a former auction, i.e., they might loose again with more or less the same probability.

It can be observed that, in the competitive approach, enhancement tends to balances the budget of the vehicles at the front of the lane, leading to less balance in waiting times: the same vehicle might lose several auctions in a row because its bids are similar to the other vehicle's ones, even if its budget is not, but because of the effect of the enhancement. We observe this phenomenon in three over four cases (COMP+AP 30 vehicles, COMP+OWP 30 vehicles, $\mathrm{COMP}+\mathrm{AP} 75$ vehicles); the fourth case (COMP+OWP 75 vehicles) has a different behaviour that is worth further exploring. In the cooperative strategies, the enhancement tends to balance the times, as can be seen in all four cases, and makes the maximum average waiting times slightly smaller than in the cases in which enhancement is not used.

Notice that standard deviations for cooperative approach scenarios are really small (always in the range $[0.4,0.6]$ ), confirming the fact that this approach is fairer than the competitive one and that other choices do not seem to matter so much.

4.3.2 Average waiting time in line. Statistics are reported in Figure 5. As we can see, and as it was expected, line average waiting times with heavy traffic are more than four times that with light traffic (while the number of vehicles only doubled). Indeed, for 30 vehicles average times range between 8.9 and 12.1 time steps, while for 75 vehicles between 46.7 and 61.4. Again there are no great differences among the different cooperative approaches (average ranging between 8.9 and 9.9 for 30 vehicles and 46.7 and 51.8 


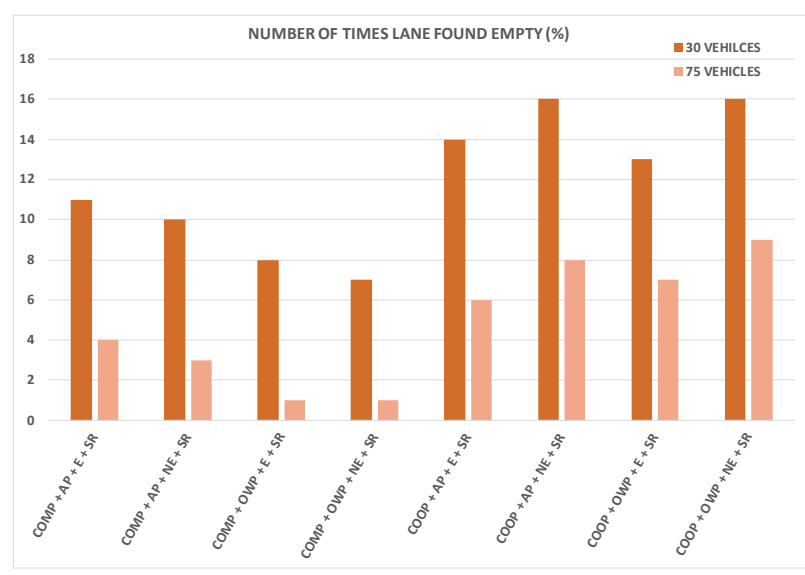

Figure 6: \% of times a lane was found empty by vehicles

for 75), with a slight improvement in the cases with enhancement and minimum average time using the most reasonable AP paying method. As for the competitive approaches, we have again very similar average times (in between 10.5 and 12.1 for cooperative, and 46.7 and 61.4 for competitive) among different combinations, but we have smaller maximum and standard deviation for the (not reasonable) AP method.

4.3.3 Zero line waiting time. Figure 6 reports the number of times vehicles find the lane empty, reported as the percentage over the total number of crossing traversals, representing the cases in which they can proceed to the auction with no further waiting. We can see that percentages are generally larger for the cooperative approach than for the competitive approach, and larger for lighter traffic than with heavier traffic. In fact, it ranges from $13 \%$ to $16 \%$ for 30 vehicles and from $6 \%$ to $9 \%$ for 75 vehicles in the cooperative case, ranging form $7 \%$ to $11 \%$ for 30 vehicles and from $1 \%$ to $4 \%$ for 75 vehicles in the competitive case. We can further observe that the enhancement has some small positive effect in the competitive approach, slightly increasing the percentage up of at most $1 \%$, while for the cooperative approach we have the opposite situation, with enhancement lowering the percentage down of at most $3 \%$.

4.3.4 Summary of results. Summing up, when imposing that all vehicles behave according to the same scenario and bids are random, what clearly emerges is that the cooperative approach presents average waiting times, for clearing both crossings and lines, smaller than the ones of the competitive approach, in all situations. This is mainly due to its round robin nature that guarantees that each vehicle at the beginning of a lane is going to get through the intersection in the auction round, and there is neither risk of delay due to aggressive bidding from other lanes nor of starvation. Moreover, the most intuitive selections for the other options (i.e., AP and enhancement enabled) give the best results (even if only slightly better than others). A competitive strategy is then not suitable to have low average waiting times for all vehicles, but we think that such an approach might be exploited only for specific situations, such as to grant priority to public transportation or public services during rush hours. Indeed, maximum line waiting times in the cooperative approach might be higher than the maximum in specific scenarios with the competitive approach. Therefore, we are planning to investigate scenarios in which vehicles behave according to different approaches in the future.

\section{CONCLUSIONS}

In this paper we presented a crossing management system for autonomous and human-driven vehicles, based on auctions. Differently from previous approaches, we have considered also humandriven vehicles, we have compared two different strategies (competitive and cooperative) and have introduced an enhancement to take into consideration also the other vehicles in the lanes, not only the first one. We performed a preliminary investigation with simple choices and results show that the cooperative approach keeps average waiting times low for all vehicles, while the competitive one leads to a much larger variability of average waiting times.

With regard to future work, we think there are several interesting directions to investigate, including: (i) experiments with more complex and larger maps, and larger and variable number of cars; (ii) quantitative comparison with other existing approaches; (iii) exploration of an adaptive approach that can adopt different strategies depending on external conditions, such as time of the day, weather condition, traffic condition, and so on; (iv) evaluation of the impact of bids "sponsored" by the vehicles in the lane behind the first vehicle to increase its chances to pass the crossing.

\section{ACKNOWLEDGMENT}

This work was supported by the EU H2020 program under the CLASS project, grant No. 780622.

\section{REFERENCES}

[1] D. Bergemann and M. Said. 2010. Dynamic auctions. Wiley Encyclopedia of Operations Research and Management Science (2010).

[2] M. Bertogna, P. Burgio, G. Cabri, and N. Capodieci. 2017. Adaptive coordination in autonomous driving: motivations and perspectives. In IEEE Int. Conf. on Enabling Technologies: Infrastructure for Collaborative Enterprises (WETICE). IEEE, 15-17.

[3] N. Capodieci, G. Andrea Pagani, G. Cabri, and M. Aiello. 2011. Smart Meter Aware Domestic Energy Trading Agents. In Proc. of the 2011 Workshop on Eenergy Market Challenge (IEEMC '11). ACM, New York, NY, USA, 1-10.

[4] D. Carlino, S. D. Boyles, and P. Stone. 2013. Auction-based autonomous intersection management. In Int. IEEE Conf. on Intelligent Transportation Systems. IEEE, 529-534.

[5] W. Chen, R. K Guha, T. J. Kwon, J. Lee, and Y-Y Hsu. 2011. A survey and challenges in routing and data dissemination in vehicular ad hoc networks. Wireless Communications and Mobile Computing 11, 7 (2011), 787-795.

[6] M. Furini, F. Mandreoli, R. Martoglia, and M. Montangero. 2017. IoT: Science Fiction or Real Revolution?. In Proc. GOODTECHS 2016. Springer International Publishing, 96-105.

[7] A. Melis, S. Mirri, C. Prandi, M. Prandini, P. Salomoni, and F. Callegati. 2016. Crowdsensing for smart mobility through a service-oriented architecture. In 2016 IEEE Int. Smart Cities Conference (ISC2). IEEE, 1-2.

[8] D. K. Murthy and A. Masrur. 2016. Braking in Close Following Platoons: The Law of the Weakest. In 2016 Euromicro Conference on Digital System Design (DSD). 613-620. https://doi.org/10.1109/DSD.2016.78

[9] Carlo Pinciroli and Giovanni Beltrame. 2016. Swarm-Oriented Programming of Distributed Robot Networks. Computer 49, 12 (2016), 32-41.

[10] J. Rios-Torres and A. A. Malikopoulos. 2017. A survey on the coordination of connected and automated vehicles at intersections and merging at highway onramps. IEEE Trans. on Intelligent Transportation Systems 18, 5 (2017), 1066-1077.

[11] H. Schepperle and K. Böhm. 2007. Agent-based traffic control using auctions. In Int. Workshop on Cooperative Information Agents. Springer, 119-133.

[12] M. Vasirani and S. Ossowski. 2012. A market-inspired approach for intersection management in urban road traffic networks. F. of Artificial Intelligence Research 43 (2012), 621-659.

[13] W. Vickrey. 1961. Counterspeculation, auctions, and competitive sealed tenders. The fournal of finance 16, 1 (1961), 8-37. 